\title{
ON THE PHYLOGENETIC DIFFERENTIATION OF THE ORGANS OF SMELL AND TASTE.
}

BY

\author{
C. JUDSON HERRICK.
}

(From the Anatomical Laboratory of the University of Chicago.)

There are in vertebrates two systems of sense organs adapted to respond directly to peripheral chemical excitation, the organs of smell and taste. In this respect they are in contrast with the other sense organs of the body; but when we come to compare the two chemical senses with one another we find it difficult to discover any objective difference between their stimuli or any explanation for the development of two chemical senses in the primitive aquatic vertebrates. And yet the very lowest vertebrates exhibit important morphological differences between the peripheral organs of smell and taste, a complete separateness in the nervous pathways to the brain and still more important differences in the central reflex connections within the brain. In view of the similarity in the nature of the stimuli to which the peripheral organs respond, these fundamental central differences have thus far baffled explanation.

Let us first consider briefly the criteria by which in the case of human beings the modalities of sense may be distinguished. (I) Doubtless the most important criterion for us is direct introspective experience, the psychological criterion. (2) The adequate stimuli of the various senses exhibit characteristic physical or chemical differences, the physical criterion. (3) The data of anatomy and physiology may differentiate structurally the receptive organs and conduction paths of the several types of sensation, the anatomical criterion. (4) The type of response varies in a characteristic way for the different senses, the physiological criterion.

It is impossible in the present state of our knowledge to frame adequate definitions of all of the senses in terms of any one of these criteria alone. Thus, we are not able introspectively to dis- 


\section{$15^{8}$ Journal of Comparative Neurology and Psychology.}

criminate between olfactory and gustatory sensations, but rather elaborate physiological experimentation is necessary to enable us to effect the analysis of these two sets of stimuli. Again, the anatomical and physiological bases of several of the senses are still very imperfectly known and in still other cases we are almost wholly ignorant of the distinctive chemico-physical qualities of the stimuli which call forth diverse sense modalities. The latter point is notably true for the senses of smell and taste. The common statement that we smell substances only in the gaseous state and taste liquids (solutions) is only approximately true, if at all, in the mammals, and certainly cannot hold for the lowly aquatic vertebrates where the differentiation of these two sense organs in practically their definitive form first occurred.

Attention has been drawn to the fact that, while tastes can be classified under the four subjective qualities, sweet, sour, bitter and salty, the innumerable odors are apparently quite incapable of any such classification. To this it may be added, on the one hand, that ZWAardeMAKER claims to be able to classify the known odors into some nine groups which he compares with the four classes of taste, and, on the other hand, that some recent studies on the chemical physiology of taste ${ }^{1}$ go to show that it is a reaction between the receiving organ and the ions of the sapid substances and that the ions belonging, to a given group, such as those giving "salty" tastes, do not all produce the same sensation quality. In other words, the four groups of taste qualities, like the nine groups of smell qualities, are more or less ill defined both from the standpoints of their psychological and their physico-chemical criteria. It is to be expected that future research will shed additional light on the physical and psychological criteria of smell and taste, but it will not eliminate their strong similarity.

These considerations suggest that smell and taste have originated phylogenetically from a common undifferentiated chemical sense, a conclusion which is supported by the morphological relations of their cerebral centers. The details of this anatomical evidence are far too complex to be summarized here and the reader

\footnotetext{
1L. Kahlenberg: The action of solutions on the sense of taste. Bul. Univ. Wisconsin, Science Series, vol. 2, pp. I-3I. 1898 .

T. W. Richards: The relation of the taste of acids to their degree of dissociation. Am. Chemical Fournal. 1898 .
} 
is referred to the exposition and discussion of the cerebral centers for smell and taste given by JoHNSTON and HERRICK. ${ }^{2}$

But despite these fundamental similarities, it still remains true that the organs of smell and taste are topographically widely separated and structurally very different both peripherally and centrally. Their central neural pathways and connections are in fact as different as are those for hearing and vision, two senses whose psychological and physical criteria are most clearly defined. The anatomical relations of the gustatory system are known in lower vertebrates and those of the olfactory system are well understood and are tolerably uniform throughout the vertebrate series. It is possible to determine by experiment to which one of the peripheral sense organs an animal responds when given a chemical stimulus. The anatomical criteria of smell and taste are therefore clearly defined.

As far as vertebrates are concerned, we may define taste in accordance with the anatomical criterion as the reaction or sensation arising from the appropriate chemical stimulation of the organs known as taste buds (wherever found in the body), and smell as the reaction or sensation arising from the appropriate chemical stimulation of the termini of the olfactory nerve. (See the Addendum, p. 165.)

These definitions cannot be extended to the invertebrates unless homologous organs can be discovered among them. It may well be that there are no such organs in the invertebrates, a single chemical sense alone serving their needs; or two or more chemical senses may be present among the invertebrates which are wholly unlike either of the vertebrate senses.

In this discussion it will be observed that I take a somewhat different standpoint from that of NAGEL, ${ }^{3}$ who defined taste and smell in terms of the state of physical aggregation of the stimulus. Smell, he says, is the faculty of perceiving vaporous (dampfförmige) substances and taste is the faculty of perceiving liquid substances. It follows from this, he argues, that it is not proper to attribute to aquatic animals a sense of smell in addition to a sense of taste, but both functions fuse into a single one.

\footnotetext{
${ }^{2}$ J. B. Johnston: The nervous system of vertebrates. Philadelphia, 1906, chap. Io.

C. Judson Herrick: The central gustatory paths in the brains of bony fishes. Fourn. Comp. Neurol. and Psych., vol. I 5, I905, Pp. 450-454.

\& W. A. NAGLL: Vergleichend physiologische und anatomische Untersuchungen über den Geruchsund Geschmackssinn und ihre Organe, mit einleitenden Betrachtungen aus der allgemeinen vergleicheaden Sinnesphysiologie. Bibliotheca Zaologica, Stuttgart, Heft 18. 1894.
} 
His argument for the absence of smell in all aquatic animals is based upon the definition of smell as the perception of gaseous or vaporous stimuli. He adduces evidence that when air is dissolved in water it is incapable of absorbing the vapors given off by volatile substances unless these vapors are soluble in the water itself, stating that they cannot be dissolved in the air contained in the water. They affect the organs, therefore, as true solutions, not as gases dissolved in water. He says (p. 60), "All substances which pass over into water from an object lying in the water, say a decomposing organic body, diffuse themselves in the water in accordance with the laws of the diffusion of liquids, not those of gases and vapors, even though the object in question when brought into the air may have vaporous emanations."

It is unnecessary to summarize here his elaborate argument for the absence of smell in fishes based upon anatomical differences in the receptive olfactory organs between fishes and air breathing vertebrates; for when examined closely in the light of our present knowledge these differences are seen to be trifling when compared with the broad resemblances of both peripheral and central organs of smell throughout the whole vertebrate phylum.

NAGEL's conclusion is expressed on p. 62: "We can with the greatest probability assume that the end-buds of the glossopharyngeus in the mouth of fishes and amphibians serve the chemical sense, viz: taste, and thus function in eating. We can with some probability assume that the sense organs of fishes and aquatic amphibia supplied by the $N$. olfactorius likewise serve the chemical sense; but this is certainly no olfactory organ in the sense of that term in the land animals. What the occasion of its chemical excitation may be is quite unknown. The method by which it is excited is with highest probability similar to the excitation of the taste buds in the mouth, i. e., the excitation follows through substances dissolved in water."

This conclusion, to my mind, simply illustrates the fact that it is impossible in the present state of our knowledge to interpret these two senses in terms of the physical stimuli. It is not meant to imply that there is no difference between the physical stimuli of smell and taste; for I think it probable that further research will bring such differences to light. But these differences are apparently very small in aquatic animals, whereas the structural differences between the nervous apparatus involved are very great indeed, even in the lowest fishes. 
Our argument thus far leads to an apparent impasse. The physical and psychological criteria of smell and taste seem inadequate to account for the definite and fundamentally different anatomical peculiarities of the organs in question. But we have not yet considered the fourth line of evidence mentioned at the beginning, that which we called the physiological criterion; viz: the characteristic responses normally following the stimulation of these organs of sense.

A suggestion made by Professor Sherrington in his recent Lectures on the Integrative Function of the Nervous System seems to me to put the matter in a perfectly clear light. As is well known, SHERRINGTON classifies the sense organs (receptors) into (I) exteroceptors, adapted for response to stimuli arising from without the body; (2) proprioceptors, sense organs lying within the body adapted to report to the central nervous system the physiological state of the organs of somatic response themselves (typified by muscle spindles, neuro-tendon organs, etc.); (3) interoceptors, organs set to to guard the receptive surfaces of the body--enteron, lungs, etc. Exteroceptors which are excited by stimuli arising at a distance from the body are termed by SHERrington distance receptors.

The physiological analysis here outlined is full of helpful suggestion in the morphology of the nervous system. Putting SHERRINGTON's analysis into correlation with that of the new school of functional morphologists, we recognize his first two types of receptors as falling within the somatic sensory group, for the chief organs of response (effectors) in both cases are the somatic or skeletal muscles. Sherrington's third type is the visceral sensory system, calling forth reflexes in the visceral musculature (including the specialized striated visceral muscles of the branchial arches and their derivatives in the higher vertebrates).

The taste buds lying within the mouth of vertebrates are typical interoceptors, and they with their nerves and cerebral centers are classified as specialized visceral sensory organs. They are in gnathostome vertebrates usually stimulated by food contained within the mouth and the effectors with which they are most directly connected are the visceral muscles of the jaws, gills, œsophagus, etc. In the protochordate vertebrate ancestry it is probable that there was but one chemical sense, and that feebly developed; for these animals probably did not masticate their 
food, and the undifferentiated primordial chemical sense may have been as important in determining the chemical character of the environing water as of the food eaten.

Be that as it may, with the appearance of teeth which pierce or crush the food, the organs of chemical sense within the mouth and pharynx assumed an important function as guardians of the entrance to the cesophagus, an interoceptive function which they perform in all gnathostome vertebrates-the organs of taste. Parallel with this differentiation within the mouth, the organs of chemical sense lying outside the mouth at the rostral end of the body would assume more and more importance as organs for detection of chemical differences in the surrounding water, differences resulting usually from the presence of sources of chemical alterations of the water lying outside the body of the fish. These external organs of chemical sensation in the leading segments of the body were finally aggregated as the organ of smell.

The differences in the character of the stimulus applied to these two organs may have been very slight at the beginning (and indeed may be so still); but in the case of any organism possessing the power of free locomotory movement the physiological significance of the stimulation of the two sense organs may be very different indeed. The object which acts as a stimulus to taste buds is already within the mouth. The appropriate reaction is typically a contraction of the visceral musculature of the mouth and pharynx adapted either to masticate and swallow or to eject the object, as the case may require. The somatic musculature is not necessarily brought into play. The olfactory organ, on the other hand, has become a distance receptor and the appropriate reaction is a movement, usually locomotor in type, of the somatic muscles, taking the animal toward or away from the source of the stimulus. Even though the stimuli in the two cases were identical, it is evident that the difference in the character of the response would bring into play a very different central reflex apparatus for the distance reaction from that for the mastication or swallowing reflex.

This difference between the characteristic reaction of the interoceptor and the distance receptor is in my opinion the sufficient explanation for the most important structural differences between the olfactory and gustatory systems of vertebrates. This same feature involves, it is true, a certain degree of difference between 
the physical stimuli and the psychical qualities of odors and savors, especially in the higher vertebrates; but these are in all animals quite subordinate to the type of reaction involved.

A critical examination of the central conduction paths for smell and taste supports this view of the case. The central olfactory apparatus is very constant throughout the vertebrate phylum. The organ of smell, as befits a distance receptor, is located in the leading segments and its central connections are with the extreme tip of the neural tube; indeed in all of the true vertebrates it has grown out rostrad beyond the primary neural tube, the entire rhinencephalon lying in the telencephalon, or ultra-terminal brain. The path extends from the olfactory bulb to the tuberculum olfactorium and other structures in the base of the forebrain, thence directly back to the olfactory centers in the thalamus or else first to the olfactory cerebral cortex (hippocampal formation, etc.) and then to the thalamus. The two principal olfactory centers in the thalamus lie in the epithalamus and hypothalamus respectively. Each of these thalamic centers receives in higher vertebrates olfactory tracts from both the basal and cortical olfactory centers of the forebrain; and each sends a strong tract to reach the motor centers. These tracts are the tr. habenulo-peduncularis (fasc. retroflexus or bundle of MEYNERT) and the fasciculus pedunculomammillaris (tr. mammillo-bulbaris). In lower vertebrates both of these tracts can be traced far downward into the medulla oblongata, where they come into relation directly with the motor nuclei of the cranial nerves and the evidence is that either directly or indirectly they pass still farther into the spinal cord for the somatic motor reflexes characteristic of olfactory reactions.

The central gustatory path is well known only in fishes. Here there are much more direct reflex connections with the visceral motor nuclei of the cranial nerves than the olfactory system shows, and in most fishes no important connections with somatic motor nuclei save by way of the hypothalamus and tractus mammillo-bulbaris. There are certain fishes, however, in which taste buds have been developed secondarily in the outer skin of the general body surface. Here they have been shown to function as exteroceptors ${ }^{4}$ and in these cases the central connections of the cutaneous taste

\footnotetext{
4C. Judson Herrick: The organ and sense of taste in fishes. Bul.U.S.Fish Cammisssion for Iqo2. Washington, 1904. The central gustatory path in the brains of bony fishes. Fourn. Comp. Neurol. and Psych., vol. 15, no. 5. 1905.
} 
I64 Fournal of Comparative Neurology and Psychology.

buds are very different from those of the phylogenetically older taste buds within the mouth. In the catfish and carp the primary cerebral center for all of the cutaneous taste buds is the facial lobe, from which secondary gustatory tracts of the typical sort pass out to the visceral motor centers, and in addition a direct secondary path to the funicular nuclei where these gustatory impulses are coördinated with tactile impressions from the same areas of skin. ${ }^{5}$ A single path leaves the funicular nuclei for the somatic motor centers, thus serving as a common reflex path for both tactile and gustatory impulses from the skin. In the $\operatorname{cod}^{6}$ the cutaneous taste buds effect somatic motor connections in an entirely different way, passing directly from the equivalent of the facial lobe into the fasciculus longitudinalis medialis and thence to the somatic motor nuclei, indicating that the cenogenetic connection of the taste buds which act as exteroceptors with somatic motor centers has been acquired independently in the gadoids and the Ostariophysi.

The interesting point in this connection is that within the group of teleosts taste buds, which typically in fishes act as interoceptors, have secondarily acquired exteroceptive functions, and parallel with this change a new central reflex path has been established between the primary centers of cutaneous (exteroceptive) taste and the somatic motor centers. It is probable that at a much more ancient period in the phylogeny of vertebrates an analogous differentiation took place in the primordial unspecialized chemical sensory apparatus, one part becoming a typical interóceptor (gustatory apparatus) and establishing its most direct central reflex connections with the visceral muscles of mastication, deglutition, etc., and another part becoming a typical exteroceptor (olfactory apparatus) and early establishing direct central reflex connections with somatic muscles of locomotion, eye movements, etc., in addition to the visceral motor reflexes characteristic of a visceral system.

It should be expressly stated that the claim is not made that all anatomical differences between the organs of smell and taste are explained by this principle, but only that in this way the direction

\footnotetext{
${ }^{5} \mathrm{C}$. Judson Herrick: On the centers for taste and touch in the medulla oblongata of fishes. Fourn. Comp. Neurol. and Psychol., vol. 16, no. 6. 1906.

B C. Judson Herrick: A study of the vagal lobes and funicular nuclei of the brain of the codfish. Fourn. Comp. Neuro!. and Psych., vol. 17, no. 1. 1907.
} 
of the original phylogenetic differentiation was determined and that this is still the dominant feature of the two.systems in question.

The conclusion is that the agencies which acted to produce the differentiation from each other of the senses of smell and taste are not to be sought primarily in the stimuli calling forth the reflexes, but rather in the character of the response evoked by the stimulus.

Addendum. As these pages pass through the press an abstract of the very interesting experiments of PARKER appears in the Proceedings of the American Society of Zoölogists (Science, n. s., vol. 27, no. 690, March 20, I908, p. 453). PARker has previously determined that the skin of the body of the frog and of various other aquatic animals is sensitive to chemical stimuli. Quite in accord with those results, he now finds that the same is true for the common fresh water catfish, Ameiurus. This fish possesses taste buds innervated by the nervus facialis scattered in the skin over practically the whole body surface. If the nerves supplying these taste buds on the trunk are cut, the fish no longer reacts to a bait in the normal way (by turning to snap at the bait) when it is presented to the flank of the body. Nevertheless such operated fishes are sensitive to sour, saline and alkaline solutions when applied to the skin of the trunk.

These results, together with the control experiments described, demonstrate that the spinal nerves of this teleost, like those of the frog, are sensitive to certain external chemical stimuli. The important question at once arises, are these responses to chemical stimulation of the spinal nerves transmitted by the same nerve fibers which transmit the tactile stimuli, or by some other component of the spinal nerves? We know from abundant physiological and clinical experience that the cutaneous rami of the spinal nerves of man transmit impulses which are perceived introspectively as very diverse sensation qualities (touch, temperature, etc.). There is evidence that some at least of the different functions of the sensory spinal nerves are served by anatomically different neurone systems; but whether the ability to respond to direct peripheral chemical stimulation is limited to one or more of these systems or common to all of them, further experiment alone can determine. 
Chemical irritability may prove to be more far-reaching and fundamental in nervous excitation than is commonly recognized. However this may be, two special reflex mechanisms have been very elaborately differentiated in vertebrates along quite diverse lines for precise and rapid response to special external chemical stimuli, the organs of smell and taste; and the explanation offered in the preceding pages for the phylogenetic differentiation of these two functional systems is not directly dependent upon any theory regarding the ultimate nature of the primordial undifferentiated sensory type from which they have sprung.

Professor Parker concludes the note to which we have referred with the remark, "From these experiments it is to be concluded that the sense of taste in horn-pouts is complex and involves not only the seventh nerve, but also the spinal nerves." Assent to this proposition will be readily granted only if we define the sense of taste in accordance with the "physical criterion" (see p. 157) as NAGEL does. In the opinion of the writer neither this criterion nor the "anatomical criterion" (as I have used it on p. I59) alone is adequate in the present state of our knowledge to serve as the basis for generally acceptable definitions of all of the so-called senses. Pending the extension of our knowledge in these fields, fruitless controversy may be avoided by a clear recognition of the fact that harmonious conclusions can be expected only on the basis of an explicit understanding regarding the standpoint chosen in every discussion. 